\title{
Measurement-based Energy Consumption Profiling of Mobile Radio Networks
}

\author{
Antonio Capone $^{1}$, Stefano D'Elia ${ }^{2}$, Ilario Filippini ${ }^{1}$, Marco Zangani ${ }^{2}$ \\ ${ }^{1}$ Politecnico di Milano, email: 'name.surname'@polimi.it \\ ${ }^{2}$ Vodafone, Network Engineering, email: 'name.surname'@vodafone.com
}

\begin{abstract}
The exponential growth of mobile traffic is forcing operators to increase quickly the capacity of their network and extend it with new technologies and improved topologies, such as heterogeneous layouts with small cells. However, since revenues cannot grow at the same rate of traffic, the main challenge is to manage capacity expansion with reduced costs. In addition to fixed costs for the new network infrastructures, operational costs are becoming critical, mainly for energy bill component. Moreover, the carbon footprint of mobile access networks is considered one of the largest of the whole ICT (Information and Communications Technology) sector and its reduction is fundamental for the environmental sustainability of the Internet economy. Due to these reasons, improving the energy efficiency of the access network is crucial for mobile operators. In order to do that, monitoring the energy consumption of the network components and defining models of energy profile are valuable approaches for estimating energy costs and identifying the most efficient configurations.

In this paper, we present an energy consumption monitoring system that has been designed and implemented in three different countries using separate sensors for the radio and base-band components of second, third and fourth generation systems. We also propose an energy profiling approach that simplifies the characterization of the different components and allows the estimation of the energy efficiency based on traffic statistics.
\end{abstract}

\section{INTRODUCTION}

The explosive growth of mobile data demand that has been observed in the last few years is stressing operator networks, which need to remarkably expand the capacity provided to the users. Predictions are that over the next 10 years, the traffic volume will skyrocket, growing by dozens of times, and the number of connected devices by many billions including also a large number of smart objects [1].

Facing this traffic growth, mobile operators are challenged with higher costs and constrained carbon footprint in providing more capacity. This requires mobile networks to become ever more cost- and resource- efficient. Among operational costs, energy costs are becoming a big share and this makes cost reduction and environmental sustainability two convergent objectives.

Even if ICT, and networks in particular, are generally considered a fundamental instrument to improve the energy efficiency of other economy sectors, their own impact on global warming is no longer negligible. ICT contributes with $2 \%$ of global greenhouse gas (GHG) emissions annually [2]-[4], which is a value that exceeds the GHG emission of the aviation sector [7]. Telecoms infrastructure and devices, in particular, contribute alone to at least one third of this value. As ICTs become more widely available, these percentages are likely to grow to approximately $2.8 \%$ of global emissions, by 2020 [6]. Also, ICT alone is responsible for a percentage between $2 \%$ and $10 \%$ of the world power consumption [5]. Estimated consumption of the network equipment (excluding servers in data centers) in 2007 was $22 \mathrm{GW}$. According to a predicted annual growth rate of $12 \%$ it will reach $95 \mathrm{GW}$ in 2020 [6].

It is well-known that radio access nodes are particularly energy-hungry, as their energy consumption can reach more than the $80 \%$ of the total energy in the entire access network [8]. This motivated research in the field of green wireless networking where the main goal is to develop devices, design protocols and network planning and optimization strategies that include energy efficiency aspects in the normal network operation [8]. Energy saving mechanisms are now becoming available in several commercial products.

Recent research efforts have shown that the energy efficiency of mobile networks, measured as the energy spent to serve a traffic unit, can be potentially improved with respect to current technology (i.e., first deployment of LTE networks) by more than a factor of 1000 combining together different solutions [1]. Even if this kind of results does not consider that the deployment costs of a completely new network infrastructure (mainly based on a large number of small cells) may limit the feasibility of such high efficiencies, it is clear that large savings can be achieved by operators if they carefully select the equipment and the configuration of the network portions they are expanding or renewing [9].

A rather big fraction of the improvement in energy efficiency is expected from advanced hardware technologies, which can significantly lower down the power required by different components, including the power amplifiers for the radio units and the processing of the base-band signals [10]. Advanced hardware platforms also allow to exploit sleep modes with very low energy consumption, which can be used with new system architectures to dynamically activate only the network portions necessary to serve the requested traffic [11], [12]. Moreover, new transmission technologies [13] and antenna systems characterized by a large number of elements [14] can greatly contribute to the reduction of the energy consumption.

While these advanced solutions start being incorporated 
in commercial products by manufacturers, it is crucial for operators to define simple instruments that allow monitoring the energy consumption of the live network and estimating the potential impact of new equipment and energy saving algorithms. Indeed, the actual savings achievable by new green approaches highly depends on the specific network scenario and traffic statistics, and an accurate planning of the most appropriate configuration is important.

In this paper, we present an energy consumption monitoring system that has been designed and implemented by Vodafone in 60 sites of three different countries using separate sensors for the radio and base-band components of second, third and fourth generation systems. The sites have been selected to represent a wide range of network scenarios in terms of mix of technologies and devices. Therefore, they can be used both to assess the current energy consumption levels and to evaluate possible new solutions. Based on a measurement campaign carried out on this system, we have designed an energy profiling approach that allows to simplify the characterization of the different components and to estimate the energy efficiency based on traffic statistics.

The reminder of the paper is organized as follows. In Section II we overview the power profiling approaches that have been proposed in the literature for the characterization of mobile access networks. In Section III we present the monitoring system for the energy consumption, while in Section IV we introduce the proposed profiling approach and some sample results. Finally, concluding remarks are given in Section V.

\section{Characterizing the Energy PeRFormance of MOBILE NETWORKS}

Characterizing the energy performance of mobile networks is not an easy task since it does not only depends on the performance of individual network devices, but also on the traffic distribution over space and time, the network deployment, as well as the propagation scenario [8]. Several performance metrics have been proposed for this purpose. One of the most popular one is the energy efficiency (Joule/bit) at full network capacity. This metric is appropriate for the case of networks working close to their capacity (full load), which is, however, rarely the case. It does not consider the coverage constraints that force the network to provide connectivity also in areas characterized by low loads for which an energy consumption over the area unit (like $\mathrm{W} / \mathrm{m}^{2}$ ) would be more appropriate. An extended version of the energy efficiency metric, averaged on time and space in order to include traffic variabilities over the entire network, appears a much more accurate approach [15].

Unfortunately, this requires an additional effort for the characterization of the network scenarios and the estimation of the most efficient configuration of network components and energy saving algorithms. In particular, modeling the impact of the base station (BS) internal components on the aggregate energy consumption is a necessary step in order to evaluate the effects of novel mechanisms and architectures for energy saving. The Energy Efficiency Evaluation Framework $\left(E^{3} F\right)$ proposed by the EARTH project maps the emitted RF power $\left(P_{\text {out }}\right)$ to the power supply of a BS site and underlines the relationship between the BS load and its power consumption [16]. Such a study is based on the analysis of the power consumption of various LTE BS types as of 2010. The effect of the various components of the BS transceivers is considered: Antenna Interface, Power Amplifier (PA), the small-signal RF transceiver, baseband interface, a DC-DC power supply, cooling, and AC-DC supply. Therefore, the $E^{3} F$ proposes a linear power consumption model that approximates the dependency of the BS power consumption to the cell load:

$$
P_{\text {in }}= \begin{cases}N_{T R X} P_{0}+\Delta_{p} P_{\text {out }}, & 0<P_{\text {out }} \leq P_{\text {max }} \\ N_{T R X} P_{\text {sleep }}, & P_{\text {out }}=0\end{cases}
$$

where $\Delta_{p}$ is the slope of the load-dependent power consumption, $N_{T R X}$ is the number of transceiver chains, and $P_{\max }$ is the RF output power at maximum load. Moreover, $P_{0}$ and $P_{\text {sleep }}$ indicate the power consumption at minimum non-zero load, and in sleep mode, respectively.

More recently, the model has been extended by the GreenTouch consortium [17] based on more detailed hardware profiles [18] so that it can be applied considering the evolution of hardware characteristics in the next years (till year 2020).

\section{MONITORING SYSTEM}

The system for monitoring and profiling the consumption of the radio access infrastructure is based on a network of remote monitoring probes installed by Vodafone in a subset of radio base station sites.

Vodafone has a wide network of smart meters installed in several thousands of radio base station sites, providing detailed information on energy use, helping to identify and target opportunities to reduce consumption and improving the accuracy of the energy billing. However, these traditional smart meters are able to measure the overall consumption in a radio base station site, while for a detailed measurement of the energy consumption of a radio base station, and for its correlation with network counters, a more detailed measurement is needed, in order to: i) monitor the energy consumption of each radio technology installed in the site, ii) separate the contribution of radio and base-band units, iii) track the evolution over time, and iv) correlate measures with specific configuration settings.

For such reason, since 2014, Vodafone selected 60 sites in different countries to implement a more complex solution, aimed at measuring not only the overall energy consumption on the site, but also the energy consumption of each component of the radio base station. In line with ETSI Recommendation $102706^{1}$, sensors were installed to measure the instantaneous energy absorption of each base station component.

\footnotetext{
${ }^{1}$ http://www.etsi.org
} 


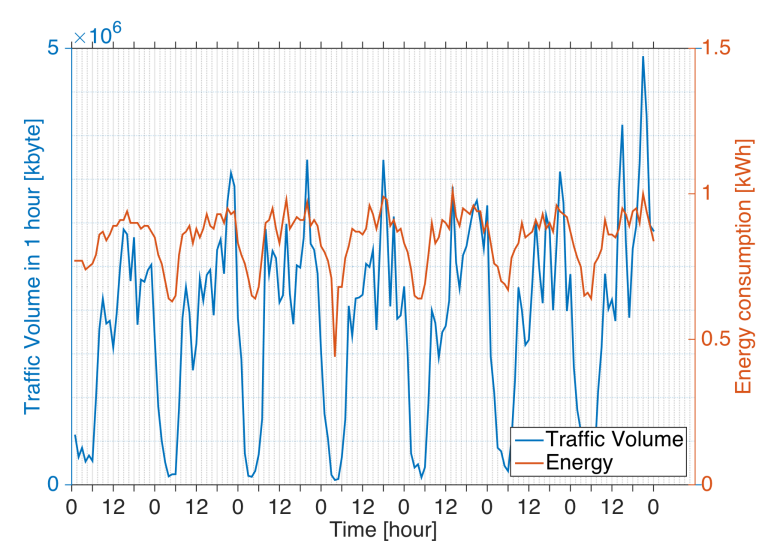

Figure 1. Time series for a 3G site where energy consumption and traffic volume are plotted together.

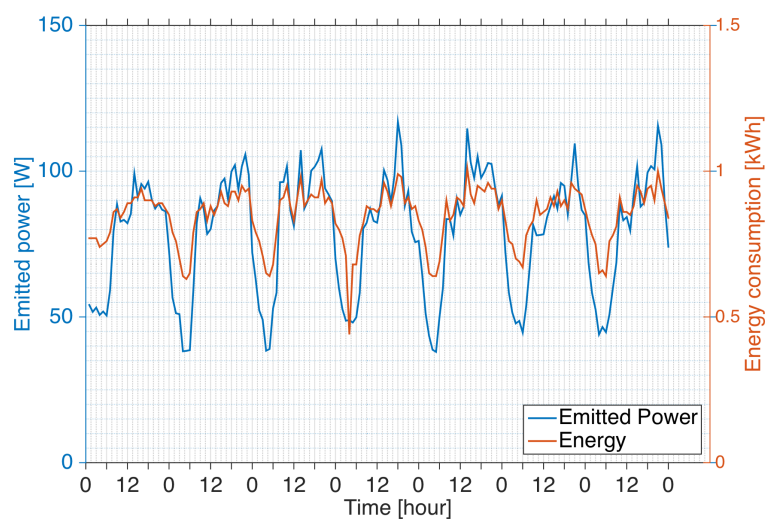

Figure 2. Time series for a 3G site where energy consumption and emitted power are plotted together.

The system implemented in each of the 60 base station sites is based on integrated current sensors (up to 16 per sites), having a size of few millimetre and its own signal microprocessor, with measurement data being transmitted digitally to a single control unit. The control unit of each site is configured to process the sensor data, supply the sensors with power, get access to the measurement data locally and transmit the data remotely. Data are then transmitted and made available into a single repository, with a time granularity that can be defined in accordance with the granularity available for network counters and key performance indicators (KPI).

\section{ENERGY PROFILING APPROACH AND EXAMPLE RESULTS}

In addition to the detailed analysis of the energy consumption of the sites where it is installed, the monitoring system implemented by Vodafone can be used to profile the energy behavior of base station components according to configuration settings and traffic variations. This can potentially provide a useful instrument for selecting the most efficient technologies and configurations also in different sites and for estimating the potential impact of new solutions on a larger portion of the network.

In order to do so, it is necessary to define energy consumption models based on variables that can be obtained

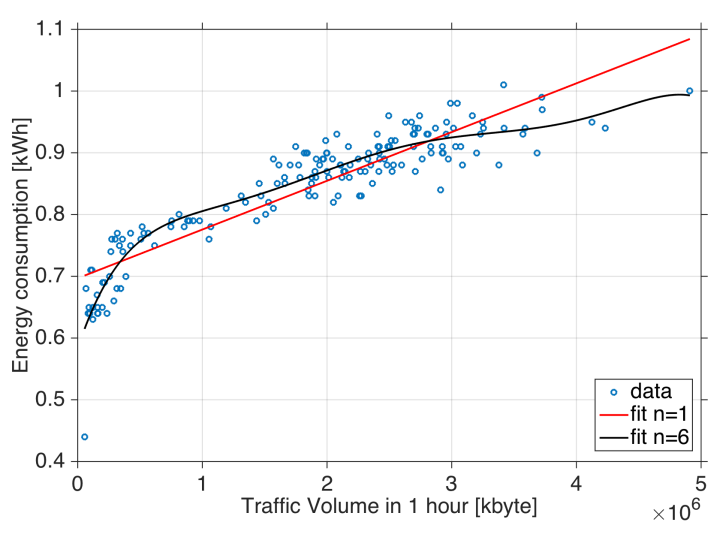

Figure 3. Energy consumption plotted against traffic volume with polynomial fitting of degrees $n=1$ and $n=6$.

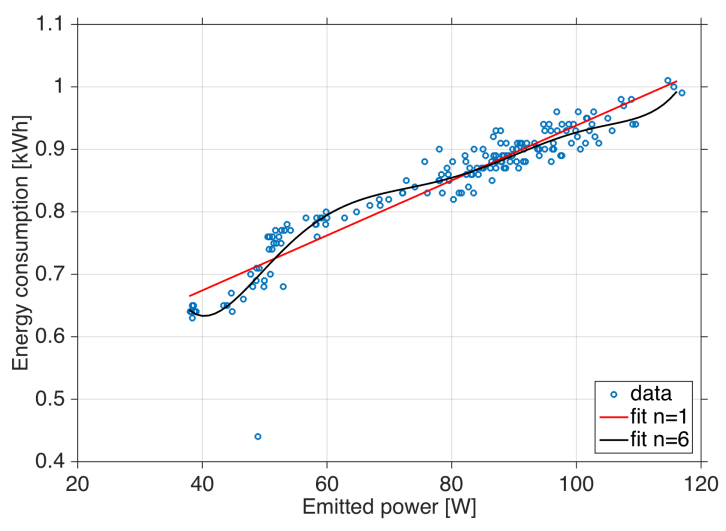

Figure 4. Energy consumption plotted against emitted power with polynomial fitting of degrees $n=1$ and $n=6$.

using network counters. We propose a simple model that extends the linear base station model based on emitted power mentioned in Section II. The model replaces the emitted power with the total user traffic transmitted in the downlink over the sampling period. Even if it is rather intuitive that the user traffic measured by network counters is directly correlated with the power emitted by base station, it is also clear that other variable elements can impact the measured energy values. Since the baseband units have an energy consumption almost constant and independent from traffic, we have focused on the radio units of different technologies. We have validated the linear model considering other more complex approaches and calculated the estimation error.

We carried out an extensive analysis over monitored sites, using traffic volume and emitted power data collected from network counter databases and compared them against energy consumption data from monitoring probes. We show below representative examples that focus on 3sector sites implementing either $3 \mathrm{G}$ or $4 \mathrm{G}$ technologies in different European countries. Each dataset consists in a week of measurements with hourly granularity.

As shown in Fig. 1 and Fig.. 2, a strong correlation exists between energy consumption and both traffic volume and emitted power. This correlation can be better appreciated in Fig. 3 and Fig. 4, where polynomial fitting curves have been tested, with degrees from 1 to 6 . 


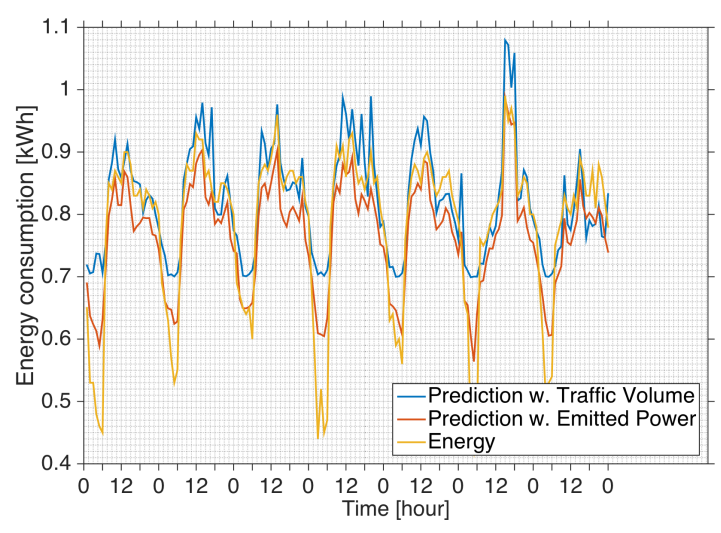

Figure 5. Example of energy consumption prediction based on both traffic volume and emitted power.

In order to validate the linear model we proceeded in the following way. We first calibrated the fitting curve, with different polynomial degrees, on a weekly training set of a single site and recorded the training error, i.e., the accuracy of the fitting. Then, we applied the calibrated curve to both the same site in different weeks, and different sites. In those tests we recorded the generalization error between the predicted energy value and the one measured by the probe, and compared it with the training error. An example of predicted energy values compared with the measured ground-truth is shown in Fig. 5.

Considering the case of training and application of the model at different weeks at the same site, Fig. 6 shows results on the accuracy of models based on both traffic volume and emitted power. As shown by dashed curves, the training error decreases by increasing the polynomial degree. This is expected, as higher-degree polynomials permit to better approximate the dataset. Note that the error is below $5 \%$ and high-degree models have only a slightly better accuracy than the linear model. In addition, models based on the emitted power have a smaller training error than ones based on traffic volume. When models are applied to datasets of different weeks, the generalization error is clearly higher than the training error, however, in all practical cases, it is below 10\%. Interestingly enough, the model based on emitted power, which predicts energy values closer to ground-truth values, exhibits a large deviation when the polynomial degree goes beyond the fourth. This is a typical behavior of fitting models, increasing the complexity to more closely follow training data leads to models with higher stiffness, that badly fit working regions outside those of the training dataset.

Similar curves are plotted in Fig. 7 when model training and application have been performed in different sites. The two sites have similar hardware features, but they are located in different environments, i.e, urban vs. rural areas. Although the effect of high-degree models is still present, we cannot conclude that the absolute value of generalization errors is always higher than those in the previous case. Indeed, there is a strong dependence on the chosen dataset. Vice versa, a true fact is that the linear model produces a generalization error of about $10 \%$.

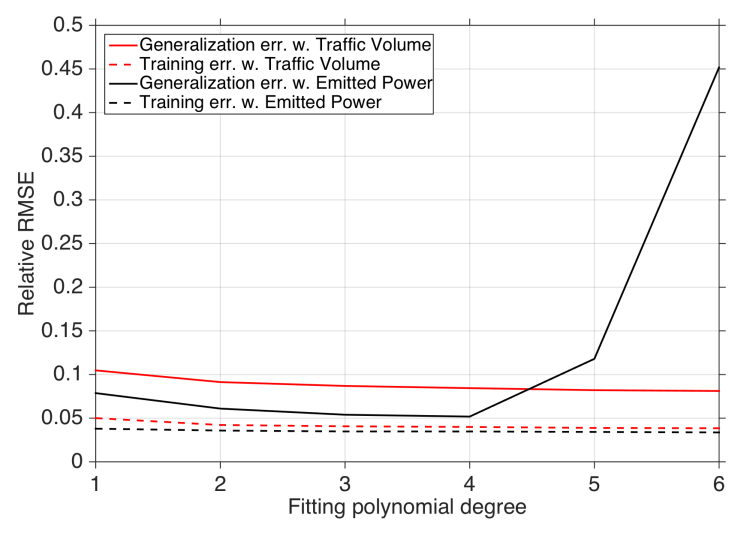

Figure 6. Training error and generalization error of different-week application varying polynomial fitting degree from $n=1$ to $n=6$.

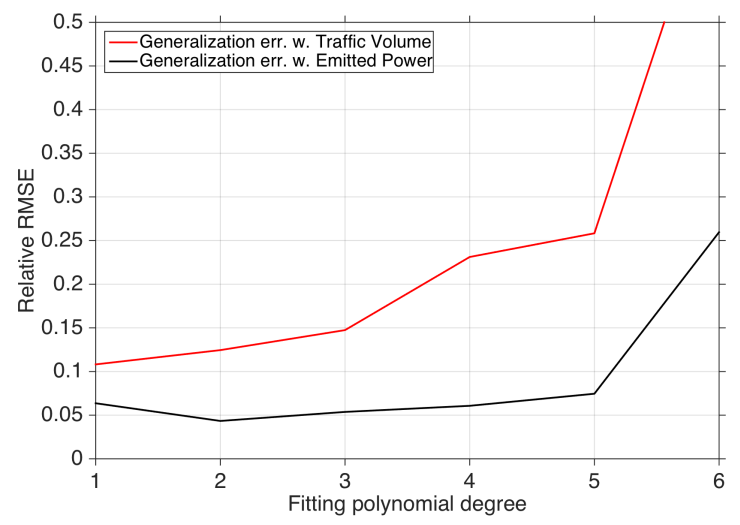

Figure 7. Generalization error of different-site application varying polynomial fitting degree from $n=1$ to $n=6$.

A general conclusion can be drawn: modeling a linear dependence between the emitted power and the energy consumption, as well as between the traffic volume and the energy consumption is a very good approximation and it is strongly confirmed by real data. In addition, the model is robust to time and place shifts, therefore, it can be applied by mobile operators to the whole network in order to predict the energy consumption relying only on network counters. This allows operators to place the energy measurement probes only in a limited set of sites and nevertheless have a complete picture of the network energy consumption. The linear model based on traffic volume, although based on simpler network counters than those related to emitted power, shows a very close prediction quality. Considering its low-complexity and its good accuracy, it represents a perfect trade-off for being implemented in live networks.

Clearly, the slope and the offset of the linear model depend on the device manufacturer and the type of technology. For instance, in Fig. 8 is shown an example of $3 \mathrm{G}$ site in a different country than that of above sites, where in-field devices come from a different manufacturer. It shows a smaller offset and a stronger dependence on traffic volume. Fig. 9 analyzes the application of the linear model to a $4 \mathrm{G}$ site as well. It exhibits a flatter profile, higher traffic volumes and lower energy consumption with respect to a $3 \mathrm{G}$ site. 


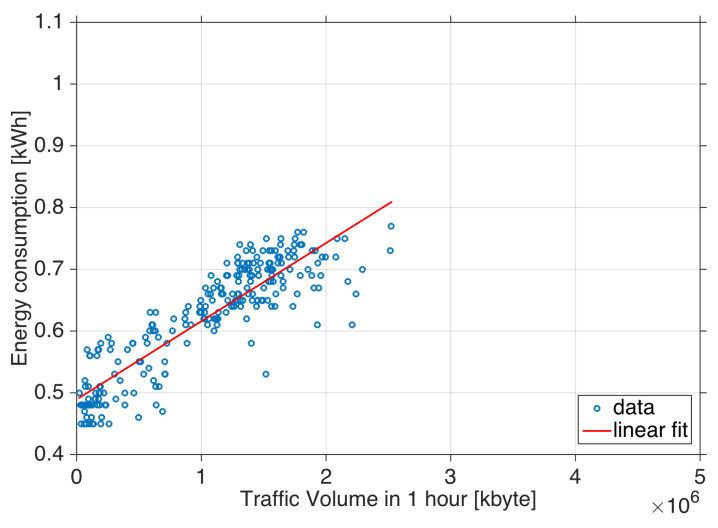

Figure 8. Energy consumption against traffic volume in a $3 \mathrm{G}$ site of a different country.

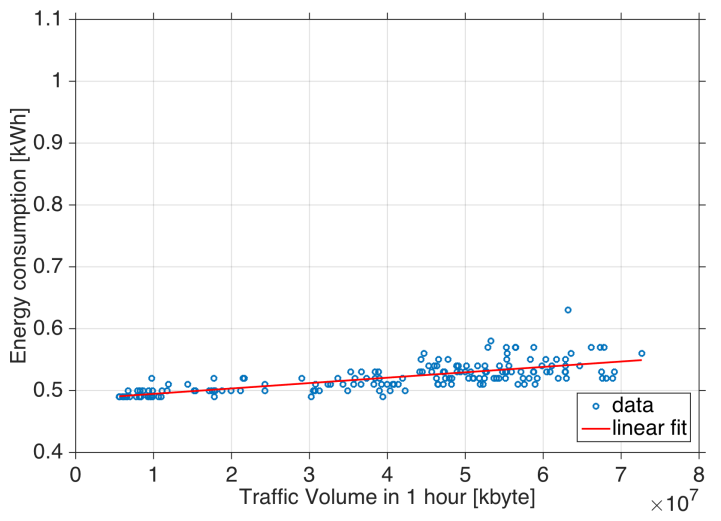

Figure 9. Energy consumption against traffic volume in a $4 \mathrm{G}$ site.

\section{Conclusion}

Operational costs are becoming a critical issue for mobile network operators due to the energy bill. Due to these reasons, monitoring the energy consumption of network components and defining energy profile models are valuable instruments to identify the most efficient network configurations and estimate energy costs.

We have presented a energy consumption monitoring system designed by Vodafone and implemented in three different countries to collect data from probes in second, third and fourth generation systems. We have analyzed data from this system and compared them with those collected by network counters in order to derive a energy profiling approach that allows to simplify the characterization of the different components and to estimate the energy efficiency based on traffic statistics.

Probes' data confirm the validity of an energy profile that models a linear dependence between the download traffic volume and the site energy consumption. The slope and the offset of the model depends on the device manufacturer and the technology generation. The model exhibits a good robustness to time and place shifts, proving, once trained on a small set of sites, its applicability to the whole network to predict the energy consumption relying only on network counters.

\section{ACKNOWLEDGMENTS}

This work has been partially supported by the Italian Ministry for Education, University and Research (MIUR) through the national cluster project SHELL, Smart Living technologies (grant number: CTN01 00128 111357).

\section{REFERENCES}

[1]

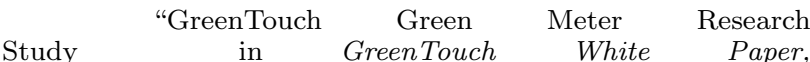
http://www.greentouch.org/uploads/documents/Green Touch Green_Meter_Research_Study_26_June_2013.pdf, June 26, 2013.

[2] H. Toure, "ICTs and climate change - the ITU perspective," in Climate Action 2008, www.climateactionprogramme.org, 2008.

[3] The Climate Group, "SMART 2020: Enabling the low carbon economy in the information age," in 2010 State of Green Business, June 2008

[4] C. Forster, I. Dickie, G. Maile, H. Smith and M. Crisp, "Understanding the environmental impact of communication systems," Tech. Rep., OFCOM, 2009.

[5] M. A. Marsan, L. Chiaraviglio, D. Ciullo and M. Meo, "Optimal energy savings in cellular access networks," in Proc. of Communications Workshops (ICC '09), IEEE, June 2009.

[6] W. Vereecken, L. Deboosere, D. Colle, B. Vermeulen, M. Pickavet, B. Dhoedt and P. Demeester, "Energy efficiency in telecommunication networks," in Proc. of the European Conference on Networks and Optical Communications (NOC '08), Krems, Austria, July 2008.

[7] A.C. Chan, A.F. Gygax, E. Wong, C.A. Leckie, Christopher, A. Nirmalathas and D.C. Kilper, "Methodologies for Assessing the Use-Phase Power Consumption and Greenhouse Gas Emissions of Telecommunications Network Services", in Environmental Science \& Technology, vol. 47, no. 1, pp. 485-492, 2013.

[8] A.D. Domenico, E.C. Strinati, A. Capone "Enabling Green Cellular Cetworks: a Survey and Outlook," Computer Communications, vol. 37, pp. 5-24, 2014.

[9] Y.Chen, O. Blume, A. Gati, A. Capone, C.-E. Wu, U. Barth, T. Marzetta, H. Zhang, S. Xu, "Energy Saving: Scaling Network Energy Efficiency Faster than Traffic Growth"," IEEE WCNC 2013 Workshop on Future gReen End-to-End wireless Network, Shanghai, China, April 7-10, 2013.

[10] C. Desset, B. Debaillie, V. Giannini, A. Fehske, G. Auer, H. Holtkamp, W. Wajda, D. Sabella, F. Richter, M.J. Gonzalez, H. Klessig, I. Godor, M. Olsson, M.A. Imran, A. Ambrosy, O. Blume., "Flexible power modeling of LTE base stations", in proc. of IEEE WCNC, Paris, France, April 2012.

[11] J. Lorincz, A. Capone, D. Begusic, "Optimized network management for energy savings of wireless access networks", Computer Networks, vol. 55, no. 3, 21 February 2011, pp. 514-540.

[12] A. Capone, I. Filippini, B. Gloss, U. Barth, "Rethinking Cellular System Architecture for Breaking Current Energy Efficiency Limits", in proc. of SustainIT 2012 (The Second IFIP Conference on Sustainable Internet and ICT for Sustainability), Pisa, Italy, Oct 4-5 2012.

[13] Yiqun Wu, Y. Chen, Jie Tang, D.K.C. So, Zhikun Xu, ChihLin I, P. Ferrand, J.M. Gorce, Chih-Hsuan Tang, Pei-Rong Li, Kai-Ten Feng, Li-Chun Wang, K. Borner, L. Thiele, "Green transmission technologies for balancing the energy efficiency and spectrum efficiency trade-off", IEEE Communications Magazine, vol.52, no.11, pp.112,120, Nov. 2014.

[14] T.L. Marzetta, "Massive MIMO: An Introduction", Bell Labs Technical Journal , vol.20, no., pp.11,22, 2015

[15] L.M.Correia, D. Zeller, O. Blume, D. Ferling, Y. Jading, I. Godor, G. Auer.L. Van der Perre, "Challenges and Enabling Technologies for Energy Aware Mobile Radio Networks", IEEE Communications Magazine, vol. 48, no. 11, Nov. 2010.

[16] G. Auer, V. Giannini, I. Godor, P. Skillermark, M. Olsson, M.A. Imran, D. Sabella, M.J. Gonzalez, C. Desset, O. Blume, A. Fehske, "How much energy is needed to run a wireless network?", IEEE Wireless Communications Magazine, vol. 18, no. 4, Oct. 2011.

[17] GreenTouch consortium, http://www.greentouch.org.

[18] C. Desset, B. Debaillie, F. Louagie, "Towards a Flexible and Future-Proof Power Model for Cellular Base Stations", in proc. of Tyrrhenian International Workshop on Digital Communications (TIWDC), Sept. 2013. 